\title{
Reconstructing Social Roles: A Grounded Theory Of Individual And Familial Coping Strategies
}

\author{
Fatemeh Ebrahimi Sadrabadi \\ Humboldt University of Berlin
}

\begin{abstract}
A body of literature supports that besides individual coping; communal coping also influences the outcomes of coping with stressful situations. Lifelong frequentative stress outcomes are often not completely restricted to individuals coping with their own issues in isolation. Thus, it is important to understand how individual and communal coping strategies function in dealing with a lifelong stressor (like chronic illness). A constructivist grounded theory approach was taken towards this study of strategies of coping with chronic illness. In-depth episodic interviews were conducted with eleven chronically ill people from ten families, comprising twenty-four family members. Through individual coping strategies, patients often simultaneously cope with their illness while also trying to protect the family from the reality of the situation. They ignore their own problems during the stable course of the illness, however, to deal with intensifying illness symptoms and complications, they begin to perform the role of a sick person (i.e., sick-role) and thereby obtain the benefits of improvisational family support. In contrast, familial coping strategy requires changing family reality through reconstruction of social roles, and establishment of the observantrole (which is the alternative to sick-role). The reconstruction of social roles in times of family stress serve to re-achieve the relative balance of family life while maintaining the hierarchy of power within the family - which is illustrated by the specific pattern of family support. Findings of this study suggest clues to the functioning of familial coping strategies in situations of lifelong frequentative family stress.
\end{abstract}

Keywords: observant-role; power; sick-role; stress; support 